\title{
A Highly Efficient P-SSHI Rectifier for Piezoelectric Energy Harvesting
}

\author{
Shaohua Lu, Student Member, IEEE, Farid Boussaid, Senior Member, IEEE
}

\begin{abstract}
A highly efficient P-SSHI based rectifier for piezoelectric energy harvesting is presented in this paper. The proposed rectifier utilizes the voltages at the two ends of the piezoelectric device (PD) to detect the polarity change of the current produced by the PD. The inversion process of the voltage across the PD is automatically controlled by diodes along the oscillating network. In contrast to prior works, the proposed rectifier exhibits several advantages in terms of efficiency, circuit simplicity, compatibility with commercially available PDs, and standalone operation. Experimental results show that the proposed rectifier can provide a $5.8 \mathrm{X}$ boost in harvested energy compared to the conventional full wave bridge rectifier.
\end{abstract}

Index Terms-Piezoelectric energy harvesting, Parallel Synchronized Switch Harvesting on Inductor, AC-DC converter.

\section{INTRODUCTION}

$\mathrm{H}$ arvesting ambient vibration energy provides a means to extend battery operation and enable self-powered ultra-low power devices, such as wireless human body health monitoring sensors or medical implants (e.g. implantable heart assists) [1-3]. A piezoelectric device (PD) (Fig. 1) vibrating at or close to its resonant frequency can be modeled as a sinusoidal current source $i_{p}$ in parallel with its internal capacitance $C_{p}$ and resistance $R_{p}$ [3]. The ac signal produced by the PD needs to be rectified for most applications. Given that the amplitude of the current $i_{p}$ generated by the PD is low (within micro-amp range) [4], the efficiency of the rectification must be as high as possible.
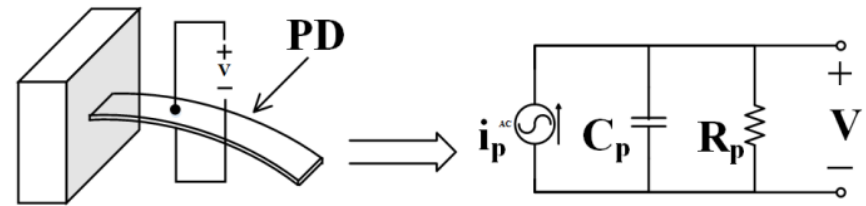

Fig. 1 Equivalent circuit of a PD vibrating around its resonant frequency

Conventional ac to dc rectification circuits for PDs include the full wave bridge rectifier (Fig. 2(a)) and the voltage doubler (Fig. 2(b)). Such circuits suffer from low efficiency due to the internal capacitance of the PD [4]. For the full wave bridge rectifier (Fig. 2(a)), the output current $i_{p}$ of the PD needs to charge (and discharge) the internal capacitance $C_{p}$ from

Shaohua Lu and Farid Boussaid are with the School of Electrical, Electronic and Computer Engineering, University of Western Australia, 35 Stirling Highway, Crawley, Western Australia 6009 (email: 10346734@student.uwa.edu.au; farid.boussaid@uwa.edu.au).
$-\left(\mathrm{V}_{\text {rect }}+2 \mathrm{~V}_{\mathrm{D}}\right)$ to $\mathrm{V}_{\text {rect }}+2 \mathrm{~V}_{\mathrm{D}}$ (or vice versa), before the current $\mathrm{i}_{\mathrm{p}}$ can actually flow to the output. This occurs every half cycle for the full wave bridge rectifier. In the case of the voltage doubler (Fig. 2(b)), the current $i_{p}$ cannot flow to the output every half cycle. This is because diode D6 is turned ON during the negative half cycle, with current $i_{p}$ flowing to ground. The shaded areas in Fig. 2 represent non-harvesting periods.

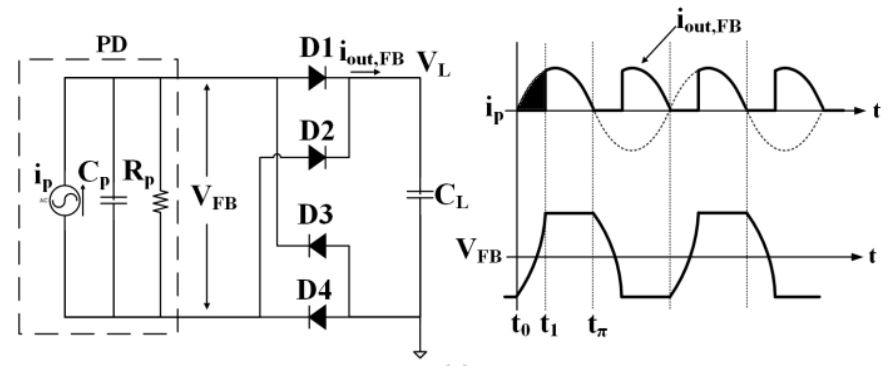

(a)

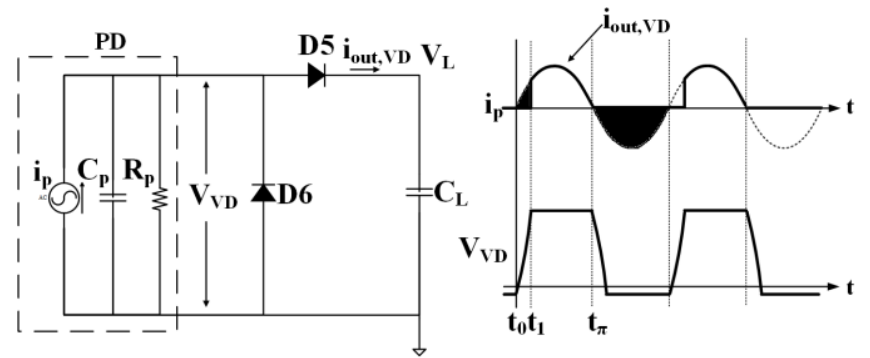

(b)

Fig. 2 (a) Full wave bridge rectifier and associated waveforms, (b) voltage doubler and associated waveforms

The PD's internal capacitance can be exploited to realize energy conversion input resonant circuits working synchronously with the vibration [5]. A well-known technique based on this approach is the Synchronized Switch Harvesting on Inductor (SSHI) technique proposed in [6]. In this technique, the PD is connected in parallel with a switch and an inductor. This technique is also called parallel SSHI (P-SSHI) (Fig. 3(a)). It operates as follows (Fig. 3(b)). At the beginning of every half cycle, the current $i_{p}$ produced by the PD changes polarity and the switch M1 is closed. As a result, the inductor L and internal capacitance $\mathrm{C}_{\mathrm{p}}$ of the $\mathrm{PD}$ forms an oscillating network and the voltage across the PD is naturally inverted. This occurs without the energy generated by the PD being used to charge/discharge the internal capacitance $\mathrm{C}_{\mathrm{p}}$. A similar technique called series-SSHI (S-SSHI) (Fig. 4(a)) was proposed in [7]. Instead of connecting the switch and inductor in parallel with the PD, the switched inductor is connected in series with the PD. The 
switch control strategy (Fig. 4(b)) is identical to that of the P-SSHI technique. When the current $i_{p}$ changes polarity, the switch M2 is closed and the energy stored in $\mathrm{C}_{\mathrm{p}}$ is transferred to the output capacitor $\mathrm{C}_{\text {rect }}$ and the voltage across the PD is inverted through the rectifier bridge. The switch $\mathrm{M} 2$ is opened at the end of the inversion process, after which M2 remains opened until the next half cycle. The analysis presented in [8] shows that the performance of P-SSHI is better than that of S-SSHI, especially when the open-circuit voltage of the PD is low. To realize the P-SSHI or S-SSHI technique, there are four main implementation challenges associated to circuit complexity, compatibility with commercially available PDs, standalone operation and harvested energy. These are: (1) detecting automatically the polarity change of the current produced by the PD. This should occur every half cycle, with a frequency of vibrations typically lower than $300 \mathrm{~Hz}$; (2) controlling automatically the switch ON time to match the duration of the inversion process. The switch $\mathrm{ON}$ time, which is of the order of microseconds, should allow for the internal capacitance $\mathrm{C}_{\mathrm{p}}$ to fully discharge into the inductor, but no longer to avoid $\mathrm{C}_{\mathrm{p}}$ taking back energy from the inductor. In addition, given that the characteristics (e.g. $\mathrm{C}_{\mathrm{p}}$ ) of the PD may not be known, the switch ON time should not be preset to allow compatibility of the rectifier with different types of PD; (3) the resistance of the oscillating network should be kept at a minimum to help improve the inversion process of the voltage across the PD; and (4) keeping the total power consumption of all circuits lower than the harvested energy to ensure self-powered operation. (a)

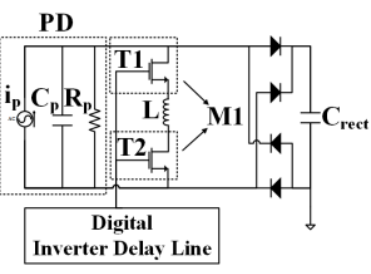

(c)

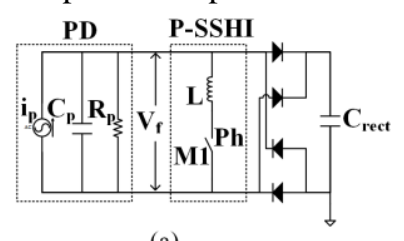

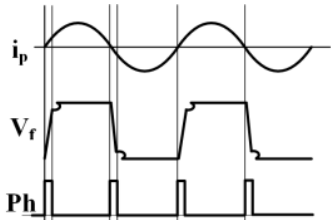

(b)

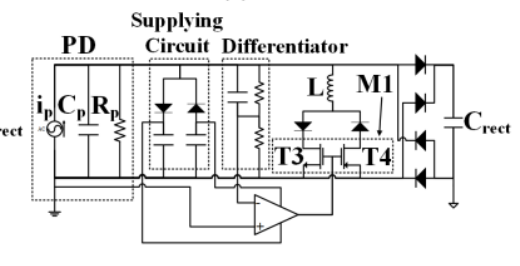

(d)

Fig. 3 (a) P-SSHI technique and (b) associated waveforms, (c) and (d) implementations of P-SSHI

[4] proposed an implementation (Fig. 3(c)) of the P-SSHI technique, where switch $\mathrm{M} 1$ is implemented using two transistors. In this implementation, switch M1's ON time is controlled by a digital inverter delay line. The latter is constructed using inverter chains including multiplexers. By applying different control words to the multiplexers, the delay line can be programmed to achieve different values of the ON time. However, the control words need to be generated externally and tuned to accommodate the internal capacitance of each specific PD. Another implementation (Fig. 3(d)) of the $\mathrm{P}-\mathrm{SSHI}$ technique was presented in [5]. The rectifier uses two

transistors (T3 and T4) and two diodes for switch M1. When the current $i_{p}$ changes polarity, the voltage signal generated by the passive differentiator changes polarity at the same time. The comparator's output turns on transistors $\mathrm{T} 3$ and $\mathrm{T} 4$ at the beginning of every half cycle. In this implementation, the comparator needs both positive and negative voltage supplies. In $[9,10]$, we proposed to use comparators and logic circuits to control the switch ON time while alleviating the need for diodes along the switching path. Given that the switch ON time is of the order of several microseconds, the rectifiers in $[9,10]$ require circuits with very fast rise/fall times and thus relatively high energy consumption compared to the harvested energy. As a result, self-power operation was thus not achieved in $[9,10]$.

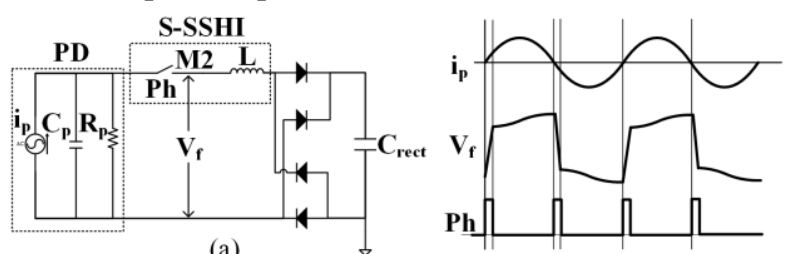

(a)

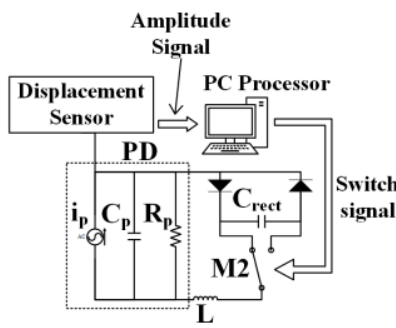

(c) (b)

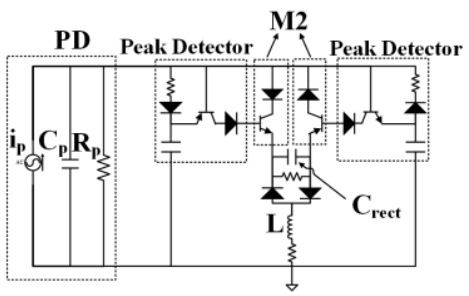

(d)

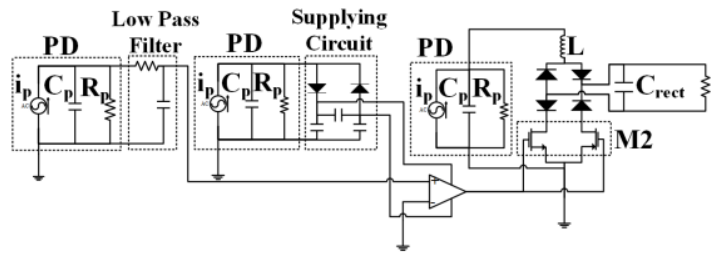

(e)

Fig. 4 (a) S-SSHI technique and (b) associated waveforms, (c), (d) and (e) implementations of S-SSHI

[9] proposed an implementation (Fig. 4(c)) of the S-SSHI technique. In this implementation, a displacement sensor and a processor are used for the synchronization and the generation of the switching commands. All of which need external power to run. $[10,11]$ implemented a self-powered S-SSHI (SP-S-SSHI) rectifier (Fig. 4(d)), which uses peak detectors to control the start of the switching time for the switch M2. However, there is always a phase lag between the peak voltage and the actual switching time. This is due to the voltage drops of diodes and transistors in the peak detector. Furthermore, this phase lag is larger for small vibrations than for large vibrations [12]. In order to eliminate the phase lag, the authors [12] designed a velocity control SSHI rectifier (Fig. 4(e)). This rectifier requires three PDs to vibrate synchronously. The first PD is used to provide power for the control circuit. The second PD is connected to a low pass filter that generates a signal to detect the polarity change of current $i_{p}$. The third PD is used for energy harvesting. This strategy results in a more complex and costly energy harvesting system. To address the above mentioned 
limitations, this paper presents a simple yet highly efficient P-SSHI based rectifier. The proposed rectifier neither relies on displacement sensor, peak detectors, differentiator or filters to detect the polarity change of the current produced by the PD, nor does it rely on a DSP or processor to generate the required signals for the control of the ON time of the switch. Furthermore, the proposed rectifier only uses the harvested energy to power the control circuits, thereby alleviating the need for any additional power supply circuits. This paper is organized as follows. Section II describes the operation of the proposed rectifier and analyses the harvested and lost energy. Section III presents the experimental setup and results. A performance comparison between the proposed rectifier and reported implementations of the SSHI technique is discussed. Section IV concludes the paper.

\section{PROPOSED RECTIFIER}

The proposed rectifier is shown in Fig. 5(a). The voltages $V_{p}$ and $V_{n}$ at the two ends of the PD are used to detect the polarity change of $i_{p}$. Before time $t_{0}, i_{p}$ is positive, $V_{p}$ is close to $\left(V_{\text {rect }}+V_{D}\right)$ and $V_{n}$ is close to but lower than $-V_{D}$, where $V_{D}$ is the diode's forward voltage. These two voltages are compared with $\mathrm{V}_{\text {ref, }}$, which is chosen slightly higher than $-\mathrm{V}_{\mathrm{D}}$. This is achieved using comparators CMP1 and CMP2. Since $V_{p}$ and $V_{n}$ are initially higher and lower than $\mathrm{V}_{\text {ref }}$ respectively, $\mathrm{OUT}_{1}$ and $\mathrm{OUT}_{2}$ are low and high accordingly. At this time, the output of the NOR gate $\mathrm{N}_{\text {out }}$ is low. When $i_{p}$ changes polarity from positive to negative at time $t_{0+}, V_{n}$ increases and reaches the value of $\mathrm{V}_{\text {ref }}$. As a result, signal OUT $\mathrm{OU}_{2}$ changes from high to low while $V_{p}$ is still higher than $V_{\text {ref }}$ and OUT ${ }_{1}$ stays low. Therefore, $\mathrm{N}_{\text {out }}$ changes from low to high. This latter change is used to detect the polarity change of $i_{p}$. When $i_{p}$ changes polarity again from negative to positive at time $t_{\pi^{+}}$, a similar process occurs. Subsequently, signal $\mathrm{N}_{\text {out }}$ is processed to generate switching commands for transistors M1-M4. When $\mathrm{i}_{\mathrm{p}}$ changes polarity from positive to negative at time $\mathrm{t}_{0+}$, the signals $\phi_{1}$ and $\phi_{\text {linv }}$ are firstly generated. As a consequence, transistors M1 and M3 are turned ON and the oscillating network Cp-L-D5-(M1, M3) is formed. Therefore, the voltage $V_{f}$ across $P D$ is naturally inverted and this inverting process spans from $t_{0}$ to $t_{1}$. Control signals $\phi_{1}$ and $\phi_{\text {linv }}$ are still high when the inverting process finishes, but diode D5 prevents the current flowing back, thereby terminating the inverting process. Subsequently, the current $i_{p}$ charges $C_{p}$ from $V_{\text {finvert }}$ to $-\left(V_{\text {rect }}+2 V_{D}\right)$ during time interval $\left[t_{1}, t_{2}\right]$ and then delivers power to the output. At time $t_{\pi+}$, when $i_{p}$ changes polarity again, a similar process occurs for oscillating network Cp-(M2, M4)-D6-L. Fig. 6 shows the schematic of the clock divider, whose input is $\mathrm{N}_{\text {out }}$ and outputs are $\phi_{1}, \phi_{\text {linv }}, \phi_{2}$ and $\phi_{2 \text { inv }}$. Input signal $\mathrm{N}_{\text {out }}$ is a sequence of pulses, toggling from low to high at the beginning of every half cycle. A Divide-by-2 circuit is used to generate two groups of control signals for the two oscillating networks. This circuit is constructed using a D flip-flop with its complementary output connected to its $\mathrm{D}$ input and signal $\mathrm{N}_{\text {out }}$ fed into the CLK input. Therefore, outputs Q and Q bar both have a frequency that is half that of signal $\mathrm{N}_{\text {out }}$. D flip-flop's output $\mathrm{Q}$ and $\mathrm{Q}$ bar are
ANDed with a delayed version of signal $\mathrm{N}_{\text {out }}$. As a result, signals $\phi_{1}$ and $\phi_{2}$ have the same pulse width than signal Nout but half its frequency. $\phi_{1 \text { inv }}$ and $\phi_{2 \text { inv }}$ are the inverted versions of $\phi_{1}$ and $\phi_{2}$.

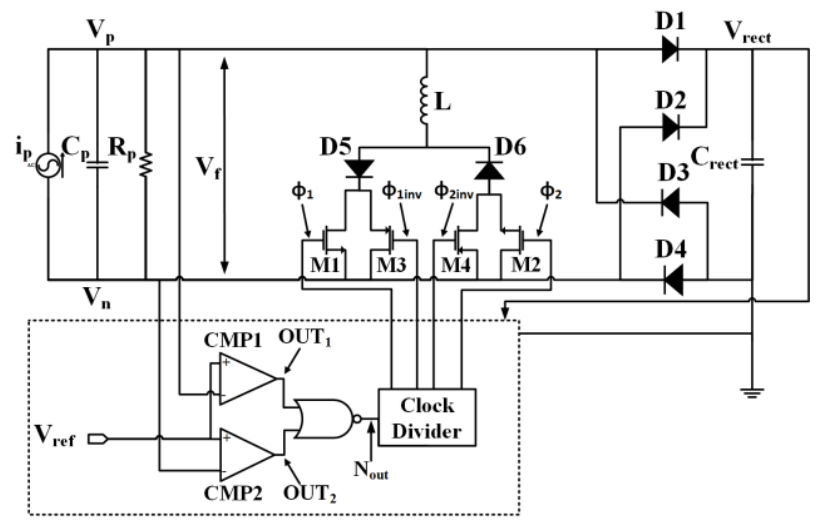

(a)

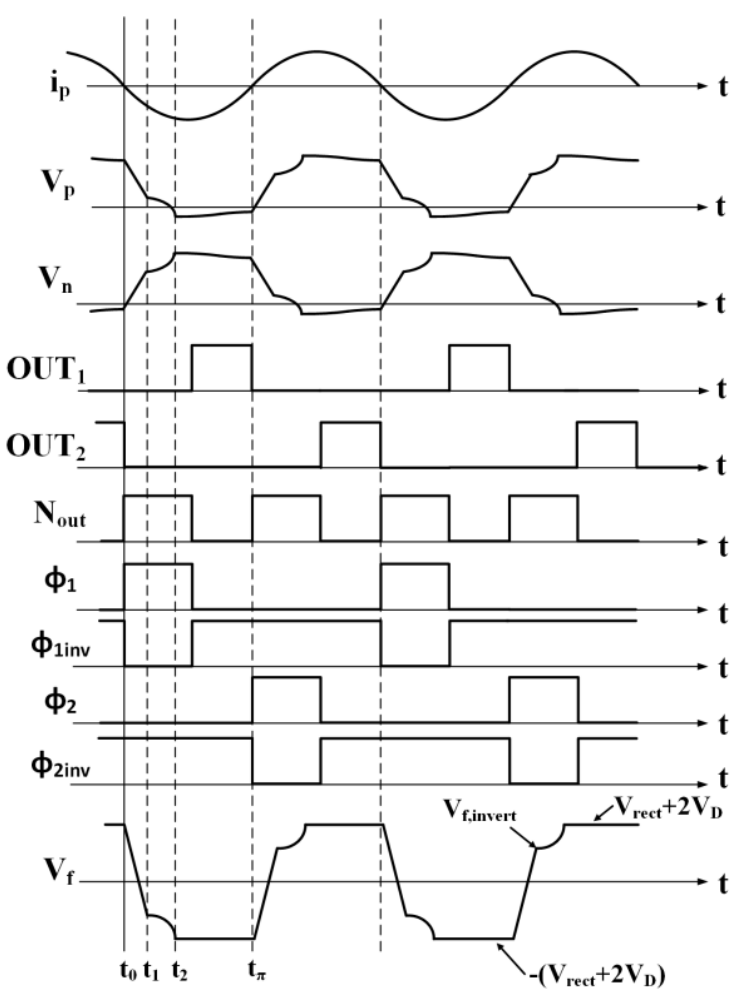

(b)

Fig. 5 (a) Implementation of the proposed rectifier and (b) associated current and voltage waveforms

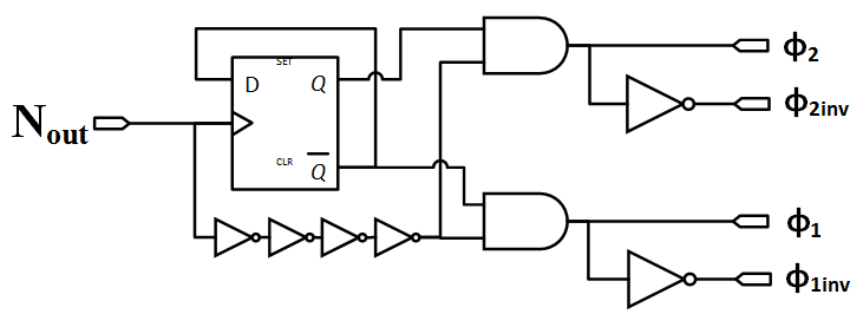

Fig. 6 Clock divider

As it can be seen from Fig. 4(b), the PD charges its internal capacitance $C_{p}$ from $V_{f, \text { invert }}$ to $\left(V_{\text {rect }}+2 V_{D}\right)$ or $-V_{f, \text { invert to }}$ 
$-\left(\mathrm{V}_{\text {rect }}+2 \mathrm{~V}_{\mathrm{D}}\right)$. Therefore, the charge lost on $\mathrm{C}_{\mathrm{p}}$ in the time interval $\left[\mathrm{t}_{1}, \mathrm{t}_{2}\right]$ is:

$$
Q_{C p, \text { loss }}=\left(V_{\text {rect }}+2 V_{D}-V_{f, \text { invert }}\right) C_{p}
$$

where $V_{f \text {,invert }}$ is the inverted voltage $V_{f}$ at the beginning of every half cycle and is given by

$$
V_{f, \text { invert }}=\left(V_{\text {rect }}+2 V_{D}\right) e^{-\frac{\pi}{Q}}-V_{D}\left(1+e^{-\frac{\pi}{Q}}\right)
$$

$\mathrm{Q}$ is the quality factor of the oscillating network and is given by:

$$
\mathrm{Q}=\frac{\omega}{\alpha}
$$

where $\omega=\sqrt{\omega_{o}^{2}-\alpha^{2}}, \omega_{o}=\frac{1}{\sqrt{L C_{p}}}$ and $\alpha=\frac{R_{\text {para }}}{2 L}, \mathrm{R}_{\text {para }}$ is the parasitic resistance of the oscillating network.

Hence the charge lost on the $\mathrm{C}_{\mathrm{p}}$ every half cycle is:

$$
\begin{aligned}
Q_{C p, \text { loss }}=\left(V_{\text {rect }}\right. & \left.+2 V_{D}\right)\left(1-e^{-\frac{\pi}{Q}}\right) C_{p} \\
& +V_{D}\left(1+e^{-\frac{\pi}{Q}}\right) C_{p}
\end{aligned}
$$

The charge lost on the internal resistance $R_{p}$ every half cycle is

$$
Q_{R p, l o s s}=\int_{t_{0}}^{t_{\pi}} \frac{V_{f}}{R_{p}} d t
$$

In time interval $\left[\mathrm{t}_{0}, \mathrm{t}_{1}\right], \mathrm{V}_{\mathrm{f}}$ is inverted by the oscillating network and the length of this time interval is given by

$$
t_{1}-t_{0}=\pi \sqrt{L C_{p}}
$$

Since the time interval $\left[\mathrm{t}_{0}, \mathrm{t}_{1}\right]$ is very short compared to the half cycle of current $i_{p}$, the energy lost in this time interval can be neglected. Then, in time interval $\left[t_{1}, t_{2}\right]$, the charge lost on $R_{p}$ is:

$$
Q_{R p, l o s s 1}=\int_{t_{1}}^{t_{2}} \frac{V_{f, t_{1}-t_{2}}}{R_{p}} d t
$$

and in this time interval, $\mathrm{V}_{\mathrm{f}}$ is given by:

$$
\begin{aligned}
V_{f, t_{1}-t_{2}} & =\frac{1}{C_{p}} \int_{t_{1}}^{t} I_{p} \sin \omega t d t+V_{f}\left(t_{1}\right) \\
& =\frac{I_{p}}{\omega C_{p}}\left(\cos \omega t_{1}-\cos \omega t\right)+V_{f}\left(t_{1}\right)
\end{aligned}
$$

The boundary condition for $\mathrm{V}_{\mathrm{f}}$ is $V_{f}\left(t_{1}\right)=\left(V_{\text {rect }}+2 V_{D}\right) e^{-\frac{\pi}{Q}}$ and $\omega \mathrm{t}_{1} \approx 0 . \mathrm{V}_{\mathrm{p}}$ is the open circuit voltage of $\mathrm{PD}$, defined as

$$
V_{p}=\frac{I_{p}}{\omega C_{p}}
$$

hence,

$$
V_{f, t_{1}-t_{2}}=V_{p}(1-\cos \omega t)+\left(V_{\text {rect }}+2 V_{D}\right) e^{-\frac{\pi}{Q}}
$$

Bringing (10) back to (7), we obtain:

$$
\begin{aligned}
Q_{R p, l o s s 1} & =\int_{t_{1}}^{t_{2}} \frac{V_{f, t_{1}-t_{2}}}{R_{p}} d t \\
& =\frac{V_{p}\left(\omega t_{2}-\sin \omega t_{2}\right)}{\omega R_{p}}+\frac{\left(V_{\text {rect }}+2 V_{D}\right) e^{-\frac{\pi}{Q}}}{R_{p}}
\end{aligned}
$$

where

$$
\omega t_{2}=\cos ^{-1}\left(1-\frac{\left(V_{\text {rect }}+2 V_{D}\right)\left(1-e^{-\frac{\pi}{Q}}\right)}{V_{p}}\right)
$$

In time interval $\left[t_{2}, t_{\pi}\right]$, the charge lost on $R_{p}$ is

$$
Q_{R p, l o s s 2}=\frac{V_{\text {rect }}+2 V_{D}}{R_{p}}\left(t_{\pi}-t_{2}\right)
$$

where

$$
t_{\pi}=\frac{\pi}{\omega}
$$

The total charge produced by PD in every half cycle is:

$$
Q_{\text {total }}=2 C_{p} V_{p}=\frac{2 I_{p}}{\omega}
$$

Therefore, the harvested power for every cycle is

$$
\begin{gathered}
P_{\text {harvest }}=2 f_{p} V_{\text {rect }}\left(Q_{\text {total }}-Q_{C p, \text { loss }}-Q_{R p, \text { loss } 1}\right. \\
\left.-Q_{R p, \text { loss } 2}\right)
\end{gathered}
$$

\section{EXPERIMENTAL RESULTS AND DISCUSSION}

The performance of the proposed rectifier was evaluated (Fig. 7) using a commercially available PD of dimensions $1.4 \times 0.24 \times 0.025$ (inch) (V22B Mide Technology) mounted on a shaker (Labworks ET-126-B1). The shaker was excited at 225 $\mathrm{Hz}$ and driven by a sine wave generator (Labworks SG-135) amplified through a power amplifier (Labworks PA-138). The proposed rectifier was built using ultra-low power off-the-shelf ICs. Comparators CMP1 and CMP2 (Fig. 4(a)) were implemented using two ultra-low power ICs (LTC1540, Linear Technology, 680 nA max quiescent supply current). The NOR gate and the clock divider (Fig. 5) were implemented using Standard CD-4000 Series CMOS gates with low input current leakage. Switches along the oscillating networks were implemented using two types of MOSFETs (VN0104 and VP0104), with on resistance of $3 \Omega$ and $11 \Omega$ for a gate voltage of $5 \mathrm{~V}$, respectively. The diodes in the oscillating networks are Schottky diodes (BAT54) with a forward voltage of $0.2 \mathrm{~V}$.

Fig. 8 shows the measured voltage waveforms across the PD and the output voltage $V_{\text {rect }}$ for the proposed rectifier and full wave bridge rectifier. The measured waveforms are consistent with the operation waveforms shown in Fig. 5(b). As seen in Fig. 8(a), the inversion process occurs each time $V_{p}$ or $V_{n}$ reaches $\mathrm{V}_{\text {ref. }}$ The control circuit can thus automatically detect the starting time of the inversion process. Note that the inversion process is not perfect with $V_{f \text {,invert }}$ lower than $V_{f}$ 


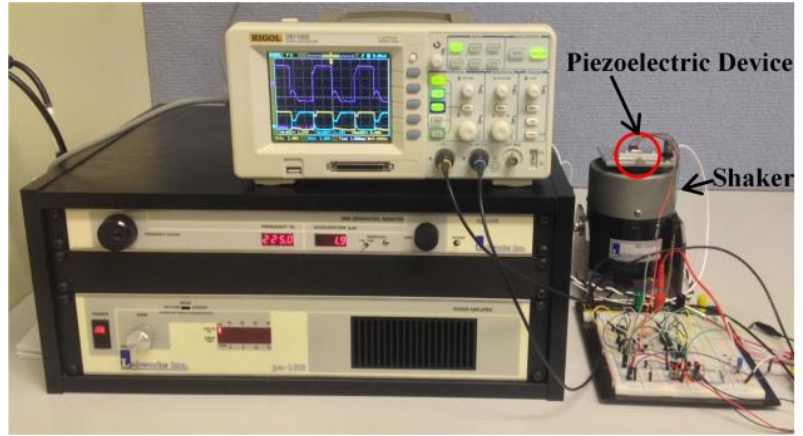

Fig. 7 Experimental Setup

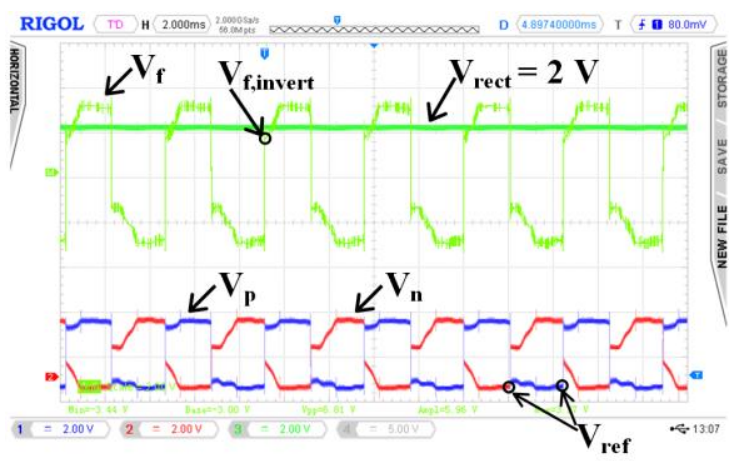

(a)

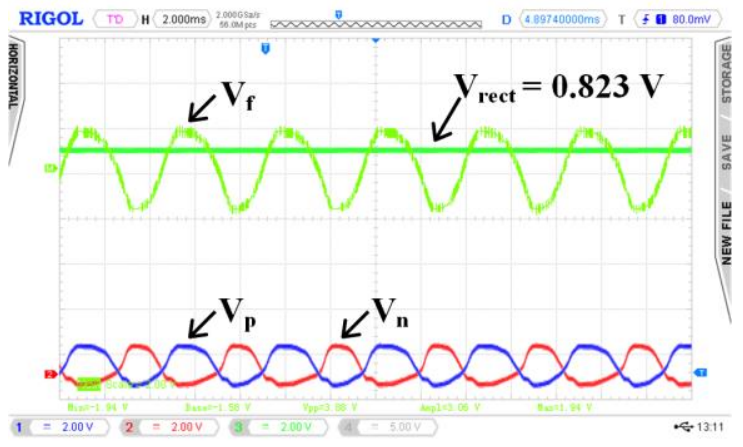

(b)

Fig. 8 Measured waveforms of voltage across the PD and output voltage $V_{\text {rect }}$ for (a) the proposed rectifier and (b) full wave bridge rectifier

because of the parasitic resistance (from inductor L and switches) along the oscillating network. In Fig. 8(a), the current $\mathrm{i}_{\mathrm{p}}$ generated by the PD only needs to charge the internal capacitance $C_{p}$ from the inverted voltage $V_{f, \text { invert }}$ to the $\pm\left(\mathrm{V}_{\text {rect }}+2 \mathrm{~V}_{\mathrm{D}}\right)$, before charges can flow to the output. A significant amount of charges have thus been saved. This can be seen by comparing the amplitudes of the voltage $\mathrm{V}_{\mathrm{f}}$ in Fig. 8(a) and Fig. $8(\mathrm{~b})$.With a $114.1 \mathrm{k} \Omega$ resistance attached to the output, the output voltage of the proposed rectifier can reach up to $2 \mathrm{~V}$. In contrast, the full wave bridge rectifier could only provide an output voltage of $0.823 \mathrm{~V}$. Fig. 9 shows the measured output power of the proposed rectifier (with different values of inductors) together with that of the full wave bridge rectifier as a function of the output voltage. The open-circuit voltage $V_{p}$ of the PD was set to $2.4 \mathrm{~V}$. The curve at the bottom of Fig. 8 with triangle symbols shows that the full wave bridge rectifier's maximum harvested power is $8.28 \mu \mathrm{W}$ for an output voltage of $0.6 \mathrm{~V}$. The middle curve with star symbols shows that the proposed rectifier (with a $22 \mu \mathrm{H}$ inductor) can provide a maximum harvested power of $20 \mu \mathrm{W}$ for an output voltage of $1.6 \mathrm{~V}$. This maximum output power is $2.45 \mathrm{X}$ that of the full wave bridge rectifier. The top curve with square symbols is the output power of the proposed rectifier with a $940 \mu \mathrm{H}$ inductor. This curve shows that the maximum harvested power of the proposed rectifier is $48 \mu \mathrm{W}$ for an output voltage of $2.6 \mathrm{~V}$. This value is $5.8 \mathrm{X}$ that of the full wave bridge rectifier.

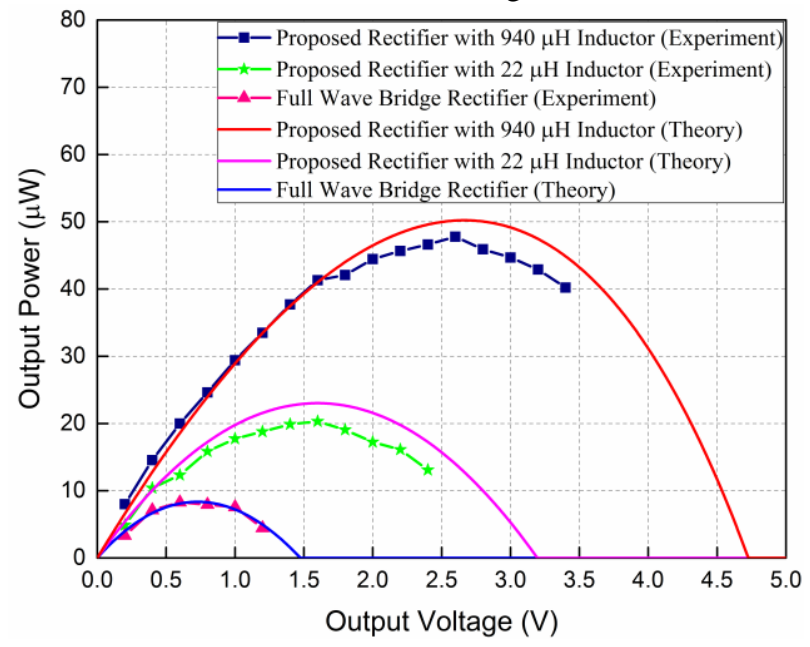

Fig. 9 Output power vs output voltage for the proposed rectifier and the full wave bridge rectifier

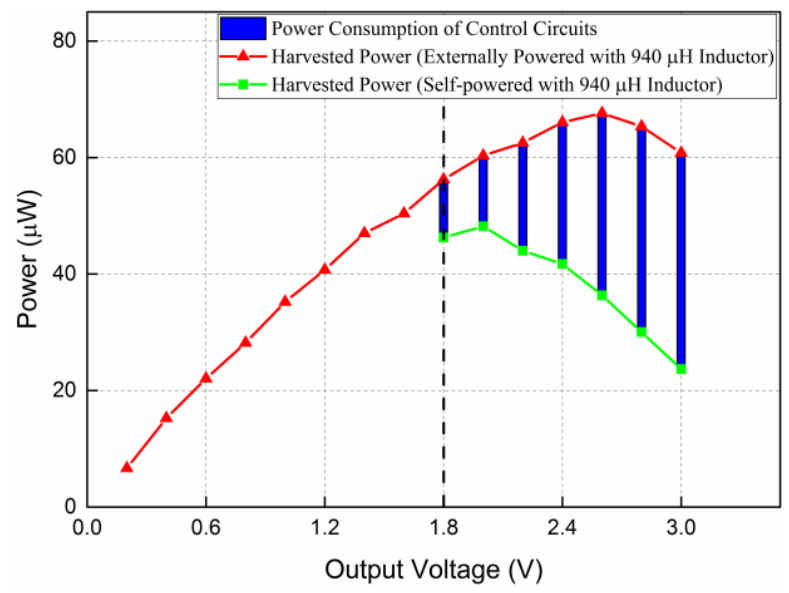

Fig. 10 Output power of externally powered and self-powered rectifiers, with power consumption of control circuits

Fig. 10 reports the output power of implemented externally powered and self-powered (by output voltage $\mathrm{V}_{\text {rect }}$ ) rectifiers together with the power consumption of control circuits. The open-circuit voltage $\mathrm{V}_{\mathrm{p}}$ of the PD was set to $3.28 \mathrm{~V}$. The output power of the self-powered rectifier is lower than that of the externally powered rectifier. The difference in the output power is the total power consumption of control circuits (blue bars in Fig. 10). When the output voltage $\mathrm{V}_{\text {rect }}$ is less than $1.8 \mathrm{~V}$, the control circuits are inactive, since this voltage is less than the minimum positive voltage supply requirement for the comparators. Table I compares the performance of the proposed rectifier against state-of-the-art SSHI implementations for piezoelectric energy harvesting. The 
second line of Table I

TABLE I - PERFORMANCE COMPARISON WITH REPORTED SSHI RECTIFIERS

\begin{tabular}{|c|c|c|c|c|c|}
\hline Publication & {$[4]$} & {$[5]$} & {$[10]$} & {$[12]$} & This Work \\
\hline Type of Implementation & Integrated & Discrete & Discrete & Discrete & Discrete \\
\hline Max Quiescent Current of Control Circuits & $>220 \mathrm{nA}$ & $4000 \mathrm{nA}$ & Not shown & $>800 \mathrm{nA}$ & $4900 \mathrm{nA}$ \\
\hline Piezoelectric Device (PD) & $\begin{array}{c}\text { V22B } \\
\text { Mide Technology }\end{array}$ & $\begin{array}{c}\text { RBL1-006 } \\
\text { Piezo System }\end{array}$ & $\begin{array}{c}\text { T120-A4E-602 } \\
\text { Piezo System }\end{array}$ & Custom Design & $\begin{array}{c}\text { V22B } \\
\text { Mide Technology }\end{array}$ \\
\hline Amplitude I of Current produced by PD & $63 \mu \mathrm{A}$ & $1.4 \mathrm{~mA}$ & $157.72 \mu \mathrm{A}$ & $88 \mu \mathrm{A}$ & $63 \mu \mathrm{A}$ \\
\hline Internal Capacitance $\mathrm{C}_{\mathrm{p}}$ & $18 \mathrm{nF}$ & $60 \mathrm{nF}$ & $33.47 \mathrm{nF}$ & $25 \mathrm{nF}$ & $18 \mathrm{nF}$ \\
\hline Vibration Frequency & $225 \mathrm{~Hz}$ & $185 \mathrm{~Hz}$ & $30 \mathrm{~Hz}$ & $12.5 \mathrm{~Hz}$ & $225 \mathrm{~Hz}$ \\
\hline $\begin{array}{c}\text { Performance compared with a Full Wave } \\
\text { Bridge Rectifier }\end{array}$ & $4 \mathrm{X}$ & $2.3 \mathrm{X}$ & $2 \mathrm{X}$ & $2 \mathrm{X}$ & $5.8 \mathrm{X}$ \\
\hline
\end{tabular}

outlines the type of implementation. Only [4] reports a CMOS integration. The advantage of such an implementation is that the quiescent current can be tailored to provide the lowest power consumption for the control circuits. All works listed in Table I rely on commercially available PDs to test the performance of the rectifiers, except [12]. The latter requires 3 PDs for the rectifier implementation, with all 3 PDs needing to vibrate synchronously. This requirement increases the complexity and cost of the whole energy harvesting system. Line 5 of Table I reports the amplitude $\mathrm{I}_{\mathrm{p}}$ of the current produced by the PD for each prior work. This amplitude depends on several factors such as the piezo material properties, vibration acceleration, PD dimensions, to name a few. Usually a PD producing a higher amplitude $I_{p}$ would generate more energy, as seen from (15). However, a higher $I_{p}$ does not necessarily translate into more harvested energy as the internal capacitance $C_{p}$ is a major source of power loss for the rectifier. A larger $C_{p}$ implies more energy loss for every half cycle of $i_{p}$, as seen from (1). This makes direct comparison with other works difficult. However, taking the conventional full wave bridge rectifier as a common reference, the proposed rectifier can improve the harvested energy by up to $5.8 \mathrm{X}$ compared to the conventional full wave bridge rectifier.

\section{CONCLUSION}

A simple yet highly efficient rectifier is proposed for piezoelectric energy harvesting. The proposed P-SSHI rectifier does not require any external signals to detect the polarity change of the current produced by the piezoelectric device (PD). Rather, it uses two comparators to monitor the voltages at the two ends of the PD to detect the polarity change of the current produced by the PD. Diodes along the oscillating network are used to terminate the inversion process. The proposed self-powered rectifier provides a significant $5.8 \mathrm{X}$ boost in terms of the harvested energy compared to the conventional full wave bridge rectifier.

\section{ACKNOWLEDGMENT}

This research was supported under Australian Research Council's Discovery Projects funding scheme (project number DP130104374).

\section{REFERENCES}

[1] N. Kong and D. S. Ha, "Low-Power Design of a Self-powered Piezoelectric Energy Harvesting System With Maximum Power Point Tracking," IEEE Transactions on Power Electronics, vol. 27, no. 5, pp. 2298-2308, 2012.

[2] G. K. Ottman, H. F. Hofmann, and G. A. Lesieutre, "Optimized piezoelectric energy harvesting circuit using step-down converter in discontinuous conduction mode," IEEE Transactions on Power Electronics, vol. 18, no. 2, pp. 696-703, Mar 2003.

[3] G. K. Ottman, H. F. Hofmann, A. C. Bhatt, and G. A. Lesieutre, "Adaptive piezoelectric energy harvesting circuit for wireless remote power supply," IEEE Transactions on Power Electronics, vol. 17, no. 5, pp. 669-676, Sep 2002.

[4] Y. Ramadass and A. Chandrakasan, "An efficient piezoelectric energy harvesting interface circuit using a bias-flip rectifier and shared inductor," IEEE Journal Solid-State Circuits, vol. 45, no. 1, pp. 189-204, January 2010.

[5] N. Krihely and S. Ben-Yaakov, "Self-Contained Resonant Rectifier for Piezoelectric Sources Under Variable Mechanical Excitation," IEEE Transactions on Power Electronics, vol. 26, no. 2, pp. 612-621, Feb 2011.

[6] D. Guyomar, A. Badel, E. Lefeuvre, and C. Richard, "Toward energy harvesting using active materials and conversion improvement by nonlinear processing," IEEE Transactions Ultrasonics, Ferroelectrics, and Frequency Control, vol. 52, no. 4, pp. 584-595, 2005.

[7] E. Lefeuvre, A. Badel, A. Benayad, L. Lebrun, C. Richard, and D. Guyomar, "A comparison between several approaches of piezoelectric energy harvesting," Journal De Physique Iv, vol. 128, no. pp. 177-186, Sep 2005.

[8] J. Dicken, P. D. Mitcheson, I. Stoianov, and E. M. Yeatman, "Power-Extraction Circuits for Piezoelectric Energy Harvesters in Miniature and Low-Power Applications," IEEE Transactions Power Electronics, vol. 27, no. 11, pp. 4514-4529, 2012.

[9] K. Makihara, J. Onoda, and T. Miyakawa, "Low energy dissipation electric circuit for energy harvesting," Smart Materials and Structures, vol. 15, no. 5, pp. 1493-1498, October 2006.

[10] J. R. Liang and W. H. Liao, "Improved Design and Analysis of Self-Powered Synchronized Switch Interface Circuit for Piezoelectric Energy Harvesting Systems," IEEE Transactions on Industrial Electronics, vol. 59, no. 4, pp. 1950-1960, Apr 2012.

[11] M. Lallart and D. Guyomar, "An optimized self-powered switching circuit for non-linear energy harvesting with low voltage output," Smart Materials \& Structures, vol. 17, no. 3, pp. 30-35, Jun 2008.

[12] C. Yu-Yin, D. Vasic, F. Costa, W. Wen-Jong, and C. K. Lee, "Self-powered piezoelectric energy harvesting device using velocity 
control synchronized switching technique," in IECON 2010 - 36th Annual Conference on IEEE Industrial Electronics Society, 2010, pp. 1785-1790. 